\title{
Wnt/ $\beta$-catenin and Hedgehog pathways are involved in the inflammatory effect of Interleukin 18 on rat chondrocytes
}

\author{
Jiapeng Bao ${ }^{1}$, Chiyuan Ma ${ }^{1}$, Jisheng Ran ${ }^{1}$, Yan Xiong ${ }^{1}$, Shigui Yan ${ }^{1}$ and Lidong $\mathbf{W u}^{1}$ \\ ${ }^{1}$ Department of Orthopedics Surgery, The 2nd Affiliated Hospital, School of Medicine, Zhejiang University, Hangzhou, 310000 \\ China \\ Correspondence to: Lidong Wu, email: wulidong@zju.edu.cn \\ Shigui Yan, email: zrjwsj@zju.edu.cn \\ Keywords: Interleukin 18; chondrocyte; osteoarthritis; Wnt/B-catenin; Hedgehog \\ Received: April 23, $2017 \quad$ Accepted: August 08, $2017 \quad$ Published: August 24, 2017 \\ Copyright: Bao et al. This is an open-access article distributed under the terms of the Creative Commons Attribution License 3.0 \\ (CC BY 3.0), which permits unrestricted use, distribution, and reproduction in any medium, provided the original author and source \\ are credited.
}

\section{ABSTRACT}

To investigate the inflammatory effect of Interleukin 18 (IL-18) on rat chondrocytes and the involvement of $\mathrm{Wnt} / \beta$-catenin and Hedgehog pathways, the mRNA and protein level of matix-degrading enzymes (MMP-2, 3, 9,13 and aggrecanses) and chondrocyte-specific proteins (Collagen II and aggrecan) were evaluated by qRT-PCR and Western blot, and key protein level of Wnt/ $\beta$-catenin and Hedgehog pathways including $\beta$-catenin, GSK-3 $\beta$, DKK-1, IHH, SHH, and Gli-2 were evaluated by Western blot. Dickkopf-1 (DKK-1) and Cyclopamine were used as antagonist of $\mathrm{Wnt} / \beta$-catenin and Hedgehog pathways to perform pathway inhibition tests. In addition, location and expression of $\beta$-catenin, GSK-3 $\beta$, Gli-2 and Smo were assessed by Immunofluorescence microscopy. The results showed up-regulation of matix-degrading enzymes (MMP-2, 3, 9,13 and aggrecanses) and down-regulation of chondrocyte-specific proteins (Collagen II and aggrecan) at both mRNA and protein level and activation of Wnt/ $\beta$-catenin and Hedgehog pathways in the inflammatory reaction on rat chondrocytes caused by IL-18 treatment was observed. As conclusion, Wnt/ $\beta$-catenin and Hedgehog pathways are involved in the inflammatory effect of IL-18 in vitro.

\section{INTRODUCTION}

Osteoarthritis (OA) is one of the most common world-wide chronic diseases [1]. A number of factors are believed to be related to the development of this potentially irreversible disease including aging, obesity, trauma, genetic disorders, systemic diseases, and other problems [2]. The development of osteoarthritis is believed to be multifactorial or resulted from a series of factors. Cartilage in joints aids in smooth mobility of joints [3]. Cartilage is consisting of chondrocytes and extracellular matrix secreted by chondrocytes which is rich in collagen and proteoglycan. Previous studies had demonstrated that the participation of immune system is one of the key elements in the development and progression of OA [4]. The production and secretion of pro-inflammatory cytokines such as tumor necrosis factor- $\alpha$ (TNF- $\alpha)$ and
Interleukin-1ß (IL-1ß) could result in the release of matrix metalloproteinases that assist in cartilage destruction [5].

Interleukin 18 is one member of the IL- 1 cytokine family, comprising 157 amino acid residues [6, 7]. IL18 could be produced by chondrocytes, osteoblasts, FLS, and macrophages in joints [8,9]. It is reported that IL-18 could increase release of MMP-1, MMP-3, and MMP13 from chondrocytes [10]. Another vitro study demonstrated that IL-18 may contribute to apoptosis in human articular chondrocytes [11]. Vivo study also indicates that IL-18 promotes joint inflammation [12, 13]. However, mechanism of how IL-18 works remains nuclear. It is reported in previous study that IL-18 induces inflammation through activation of the nuclear factor- $\mathrm{kB}$ (NF-kB), p38 mitogen activated protein kinase (MAPK) and Erk1/2 pathways [10], other pathways related to OA are poorly studied. However, it is well known that $\mathrm{Wnt} / \beta$ - 
catenin and Hedgehog pathways are another two important pathways related to OA [14-18]. DKK-1 and Cyclopamine work as potent antagonist of Wnt/ $\beta$-catenin and Hedgehog signaling $[19,20]$. Therefore, in the present study, Wnt/ $\beta$-catenin and Hedgehog pathways are investigated in inflammation reaction of chondrocytes caused by IL-18 in vitro, and our results will help understanding of the effect of IL-18 in OA.

\section{RESULTS}

Up regulation of MMP-2, 3, 9, 13 and aggrecanses (adamts 4/5), down regulation of Collagen II and aggrecan caused by IL-18 stimulation.

We performed qRT-PCR to detect the expression levels of MMP-2, 3, 9, 13, aggrecanses (adamts 4/5) Collagen II and aggrecan in chondrocytes. Chondrocytes were treated with IL-18 in different concentration $(0 \mathrm{ng} / \mathrm{ml}$ as control, $1 \mathrm{ng} / \mathrm{ml}, 10 \mathrm{ng} / \mathrm{ml}, 100 \mathrm{ng} / \mathrm{ml}$ ) for 24 hours. RTPCR showed significant up-regulation of mRNA levels of MMP-2, 3, 9, 13 adamts 4/5, down-regulation of mRNA levels of Collagen II and aggrecan, which is IL-18 dosedependent (Figure 1, Supplementary Figure 1A). Then, we assessed the protein level of MMP-2, 3, 9, 13 adamts $4 / 5$, Collagen II and aggrecan by Western blot analysis (Figure 2, Supplementary Figure 1B), which comes to the similar results of qRT-PCR. All these results suggested the inflammatory role of IL-18 on cartilage matrix by up-regulation of matrix-degrading enzymes and by downregulation of matrix components secreted chondrocytes in vitro, which is similar with the results of previous studies $[10,11]$.

IL-18 stimulation increases the levels of $\beta$-catenin, GSK-3 $\beta$, DKK-1, IHH, SHH, and Gli-2. $\beta$-catenin, GSK$3 \beta$, DKK-1 are several key proteins in $\mathrm{Wnt} / \beta$-catenin pathway, and $\mathrm{IHH}, \mathrm{SHH}$, and Gli-2 play key roles in
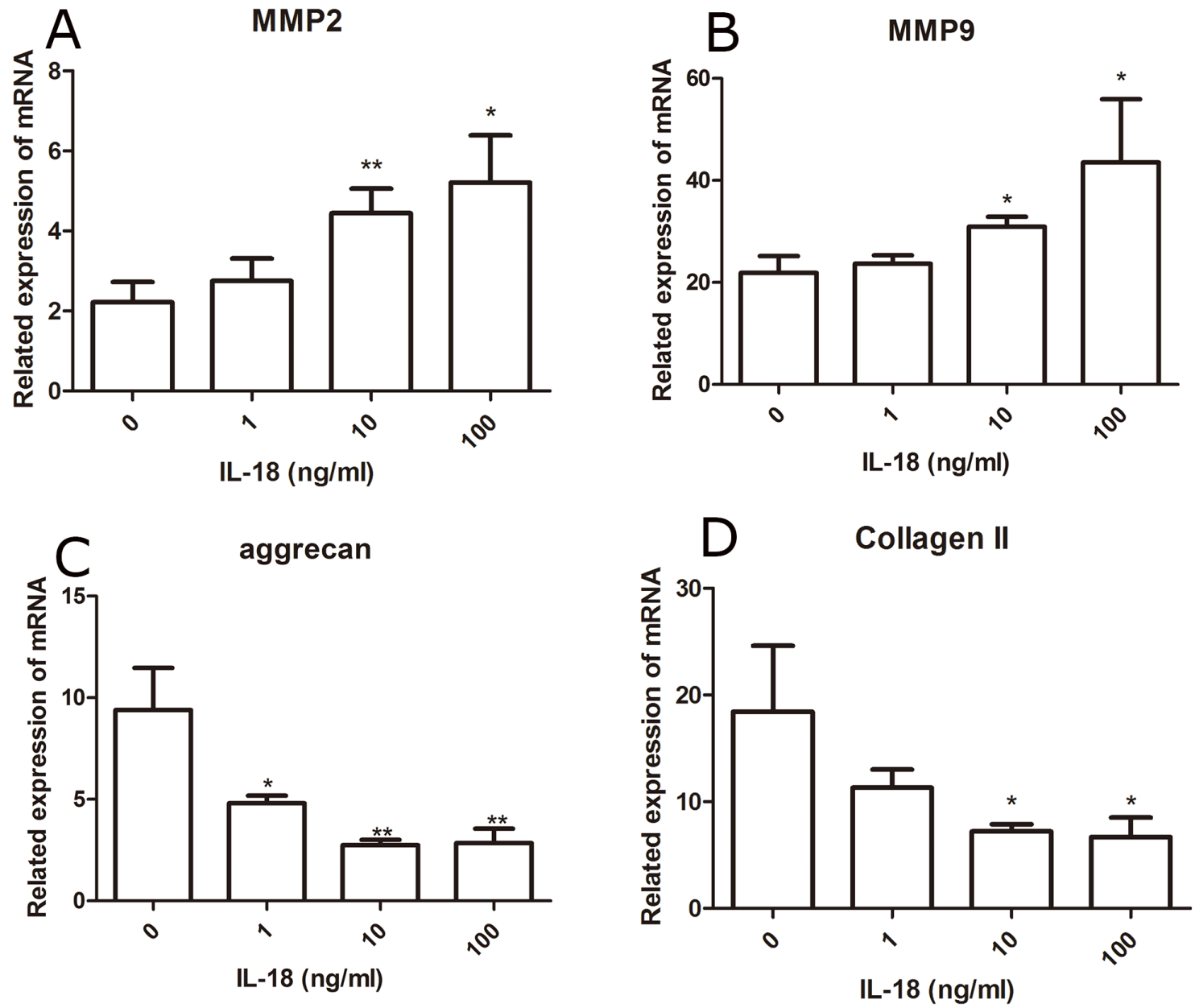

Figure 1: Up-regulation of matix-degrading enzyme genes and down-regulation of chondrocyte-specific genes at mRNA level caused by IL-18 were observed in SD rat chondrocytes. The chondrocytes were treated with different concentrations of IL-18 for 24 hours. The expression of chondrocyte-specific genes and matix-degrading enzyme genes, MMP-2 (A), MMP-9 (B), aggrecan (C), and Collagen II (D) were evaluated by Real-Time PCR. Significance was calculated by a one-way ANOVA with a post hoc Tukey's multiple comparisons test. ${ }^{*} \mathrm{p}<0.05,{ }^{* *} \mathrm{p}<0.01,{ }^{* * *} \mathrm{p}<0.001$ versus $0 \mathrm{ng} / \mathrm{ml}$ IL-18 treated group. 
Hedgehog pathway. The amounts of the proteins in cell nucleus and whole cell after $24 \mathrm{~h}$ IL-18 stimulation were investigated dividedly. The results of Western blot showed the significant increased protein level of $\beta$-catenin, GSK$3 \beta$, DKK-1, IHH, SHH, and Gli-2. $\beta$-catenin, GSK$3 \beta$ and significant decreased protein level of DKK-1 in chondrocytes treated with IL-18 at concentrations (10ng/ml, 100ng/ml) for 24 hours when compared with control, while low concentration of IL-18 (1ng/ml) treatment couldn't provide significant change (Figure 3). Furthermore, IL-18 induced nuclear translocation of $\beta$-catenin and Gli-2, which is also IL-18 dose-dependent (Figure 4). Those results suggested that IL-18 stimulation in vitro had sitimulation on the $\mathrm{Wnt} / \beta$-catenin and Hedgehog pathways and we regarded $100 \mathrm{ng} / \mathrm{ml}$ as the concentration of IL-18 stimulation in the following inhibition experiments.

The up-regulation of MMPs, aggrecanses and downregulation of Collagen II and aggrecan caused by IL-18 stimulation were partly inhibited by the treatment of DKK$1(1 \mu \mathrm{g} / \mathrm{ml})$. We performed a series of pathway inhibition tests to proof the activiation of Wnt/ $\beta$-catenin pathway. It's reported that DKK-1 works as a potent antagonist of Wnt signaling [19] and the block effect of DKK-1 was analyzed here. Immunofluorescence microscopy showed the activation of Wnt/ $\beta$-catenin pathway resulted from IL-18 stimulation was inhibited by DKK-1 treatment (Figure 5). We used qRT-PCR and Western blot analysis to assess the mRNA level and protein level of MMP-2, 3, 9, 13, adamts 4,5 , Collagen II and aggrecan changes caused by $24 \mathrm{~h}$ IL18 stimulation $(100 \mathrm{ng} / \mathrm{ml})$ after 1 hour treatment of DKK$1(1 \mu \mathrm{g} / \mathrm{ml})$ (Figure 6, Supplementary Figure 2). Results demonstrated that DKK-1 could decrease the up-regulation of MMPs, aggrecanses and down-regulation of Collagen II and aggrecan caused by IL-18 stimulation. These results indicate that IL-18 induces inflammation in chondrocytes in vitro by activating the $\mathrm{Wnt} / \beta$-catenin pathway.

The up-regulation of MMPs, aggrecanses and down-regulation of Collagen II and aggrecan caused by IL-18 stimulation were partly inhibited by the treatment of Cyclopamine $(10 \mu \mathrm{M})$. To testfy that the Hedgehog pathway is involved in the IL-18 inflammatory effect, we investigated the blocking effect of Hedgehog. It's reported that cyclopamine is a potent antagonist of Hedgehog pathway [20]. Chondrocytes were first treated with Cyclopamine $(10 \mu \mathrm{M})$ for $1 \mathrm{~h}$, and then incubated with IL-18 (100ng/ml) for $24 \mathrm{~h}$ before analysis. Immunofluorescence microscopy showed the activation of Hedgehog pathway resulted from IL-18 stimulation was inhibited by Cyclopamine treatment (Figure 7). Western
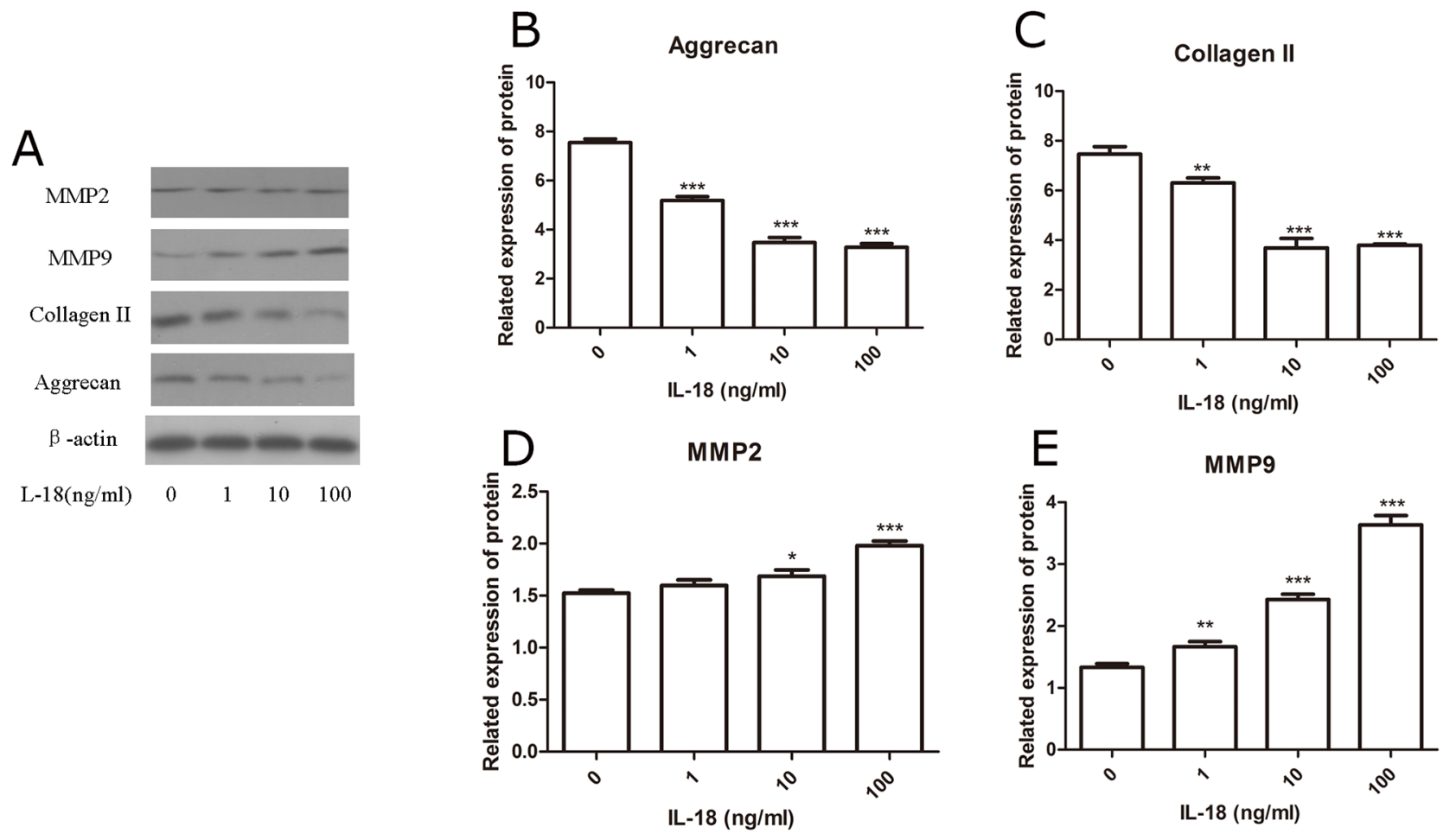

Figure 2: Up-regulation of matix-degrading enzymes and down-regulation of chondrocyte-specific proteins caused by IL-18 were observed in SD rat chondrocytes. The chondrocytes were treated with different concentrations of IL-18 for 24 hours. The expression of chondrocyte-specific proteins and matix-degrading enzymes, MMP-2 (D), MMP-9 (E), aggrecan (B), and Collagen II (C) were evaluated by Western blots (A), $\beta$-actin worked as internal control here. Significance was calculated by a one-way ANOVA with a post hoc Tukey's multiple comparisons test. ${ }^{*} \mathrm{p}<0.05,{ }^{* *} \mathrm{p}<0.01,{ }^{* * *} \mathrm{p}<0.001$ versus $0 \mathrm{ng} / \mathrm{ml}$ IL-18 treated group. 


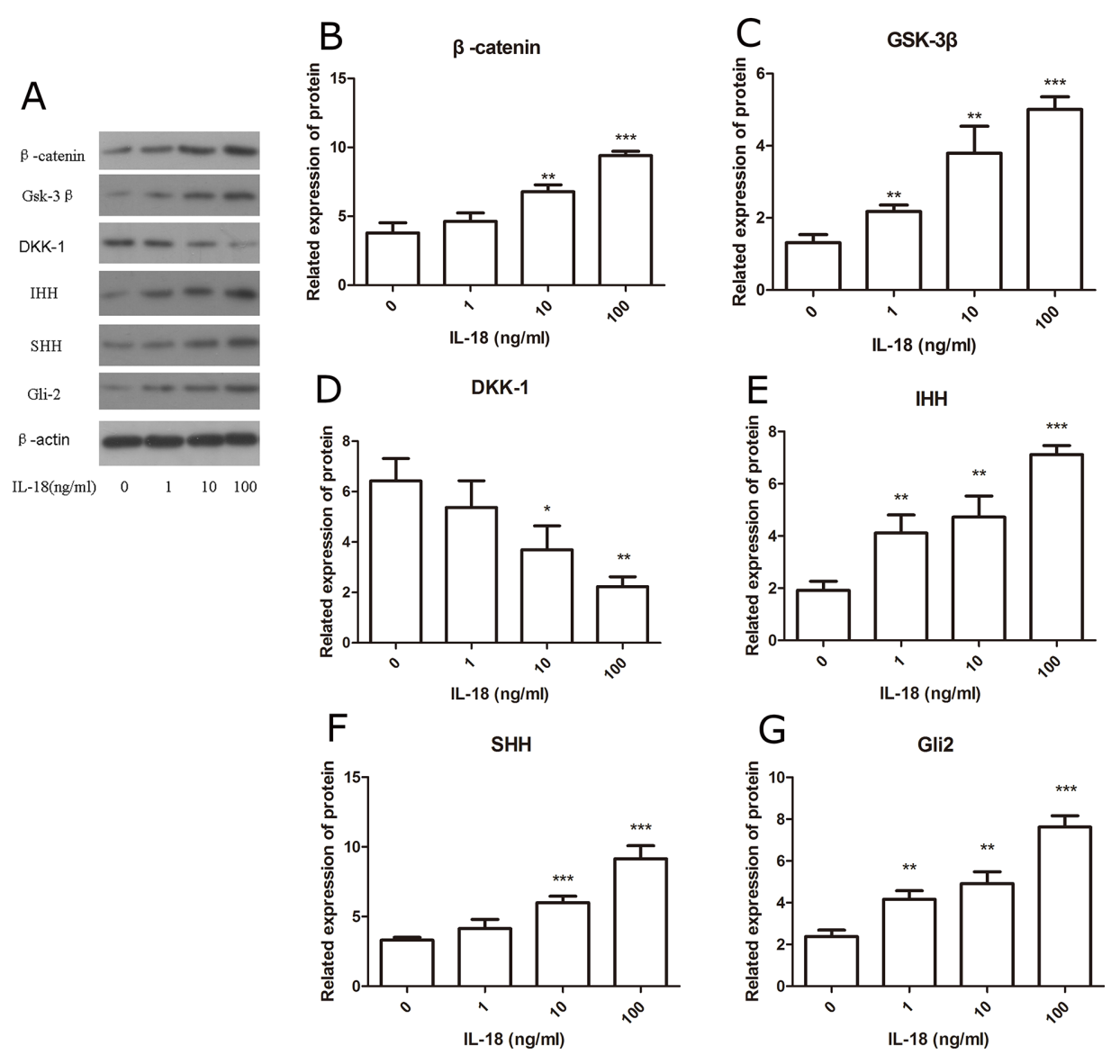

Figure 3: IL-18 stimulation increased the protein level of Wnt pathway related proteins and Hedgehog related proteins. The chondrocytes were treated with different concentrations of IL-18 for 24 hours. The level of $\beta$-catenin (B), GSK-3 $\beta$ (C), DKK-1 (D), IHH (E), SHH (F), Gli-2 (G), and $\beta$-actin in total extract was investigated by Western blot (A). Significance was calculated by a one-way ANOVA with a post hoc Tukey's multiple comparisons test. ${ }^{*} \mathrm{p}<0.05,{ }^{* *} \mathrm{p}<0.01,{ }^{* * *} \mathrm{p}<0.001$ versus $0 \mathrm{ng} / \mathrm{ml}$ IL-18 treated group.

A

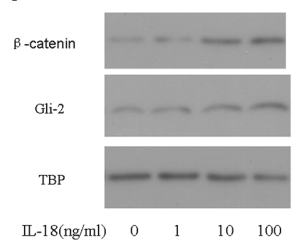

D

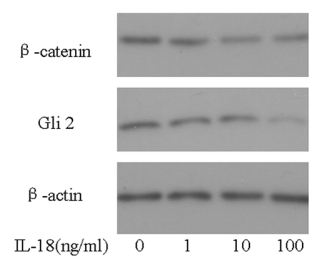

B
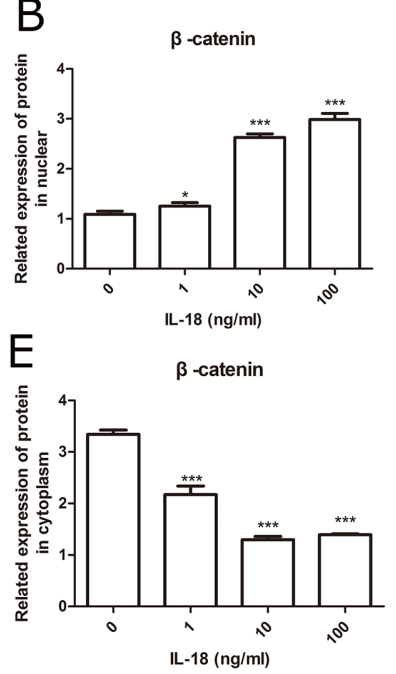

C
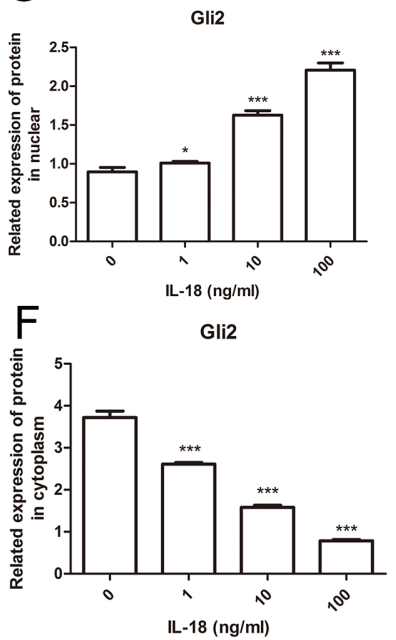

Figure 4: IL-18 induced nuclear translocation of $\beta$-catenin and Gli-2. The chondrocytes were treated with different concentrations of IL-18 for 24 hours. The level of $\beta$-catenin and Gli2 in nucleus (A-C) and cytosol (D-F) was evaluated dividedly by Western blot. Significance was calculated by a one-way ANOVA with a post hoc Tukey's multiple comparisons test. ${ }^{*} \mathrm{p}<0.05,{ }^{* *} \mathrm{p}<0.01,{ }^{* * *} \mathrm{p}<0.001$ versus 0ng/ml IL-18 treated group. 

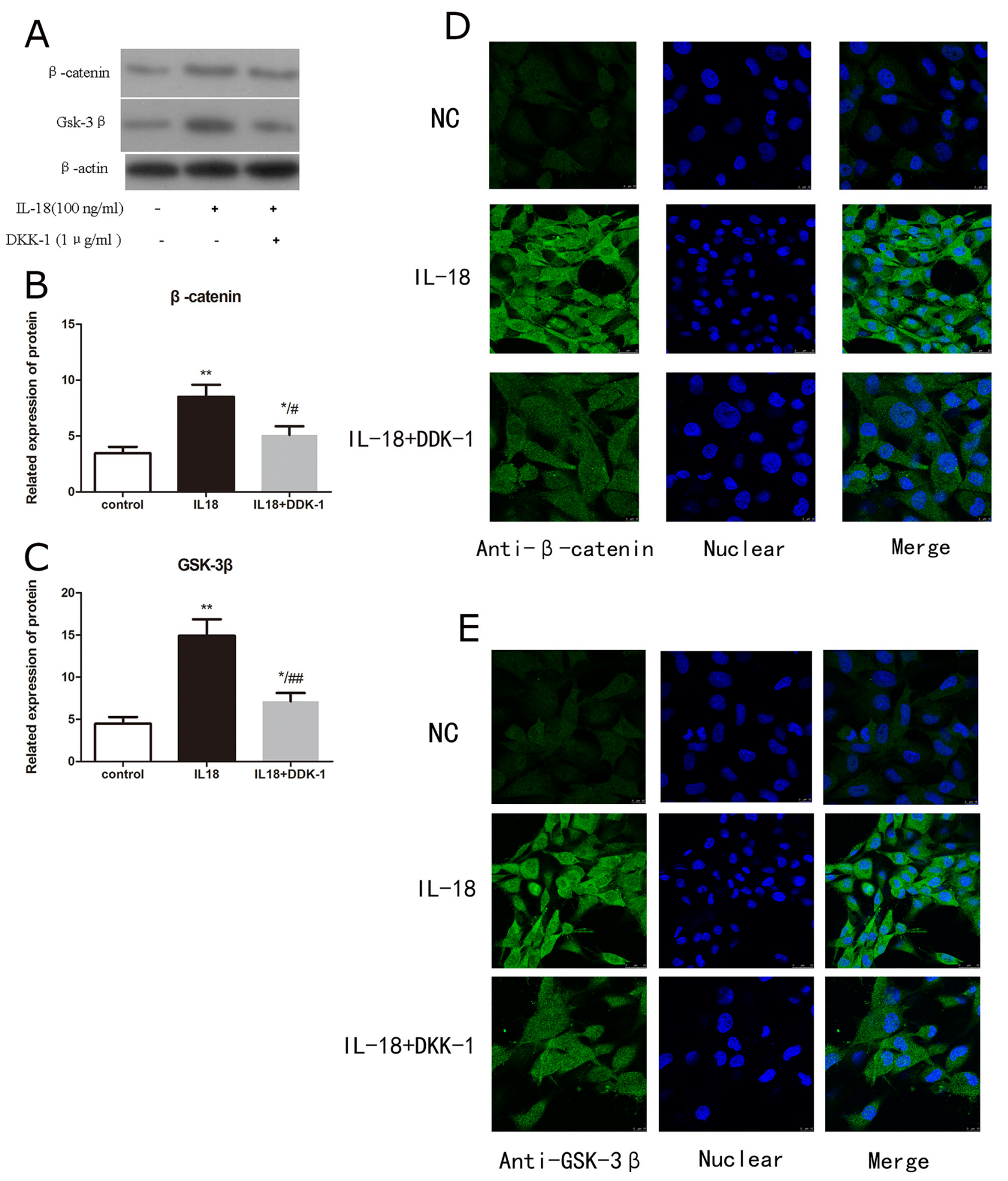

Figure 5: DKK-1 worked as a potent antagonist of Wnt signaling and the block effect of DKK-1 was analyzed here. Chondrocytes of IL-18+DKK-1 group were pre-treated with DKK-1 $(1 \mu \mathrm{g} / \mathrm{ml})$ for 1 hour, followed with $24 \mathrm{~h}$ IL-18 stimulation $(100 \mathrm{ng} / \mathrm{ml})$. Chondrocytes of IL-18 group were treated with $100 \mathrm{ng} / \mathrm{ml}$ IL-18 for 24 hours. The level of $\beta$-catenin (B) and GSK-3 $\beta$ (C) in total extract was investigated by Western blot (A). Significance was calculated by a one-way ANOVA with a post hoc Tukey's multiple comparisons test. " $\mathrm{p}<0.05,{ }^{* *} \mathrm{p}<0.01,{ }^{* * *} \mathrm{p}<0.001$ versus control group. ${ }^{*} \mathrm{p}<0.05,{ }^{\#} \mathrm{p}<0.01,{ }^{* \#+\cdots} \mathrm{p}<0.001$ versus $100 \mathrm{ng} / \mathrm{ml} \mathrm{IL}-18$ treated group. The location and concentration of $\beta$-catenin (D) and GSK-3 $\beta$ (E) was visualized using immunofluorescence analysis. 
blot and qRT-PCR were used to analyze the mRNA level and protein level of MMP-2, 3, 9, 13, adamts 4, 5, Collagen II and aggrecan changes caused by treatment. Results demonstrated that Cyclopamine could decrease the up-regulation of MMPs, aggrecanses and downregulation of Collagen II and aggrecan caused by IL-18 stimulation (Figure 8, Supplementary Figure 3), which demonstrated that Hedgehog pathway is involved in the IL-18 inflammatory effect in chondrocytes.

\section{DISCUSSION}

IL-18 is a cytokine with a series of biological actions. The up-regulated expression of IL-18 in cultured chondrocytes stimulated by IL-1 $\beta$ has been noted [8]. As a member of the IL-1 cytokine family, IL-18 is mainly produced by chondrocytes, osteoblasts, FLS, and macrophages in joints $[8,9]$, and the increased concentration of IL-18 in the synovial fluid, synovium, cartilage, and blood serum is reported to be related to the degree of severity of OA seen in radiographic images [21-23]. Several studies have confirmed the inflammatory effect of IL-18 on chondrocytes in vitro and in vivo [8, 10-12]. Furthermore, the previous study has found that the enhancement of MMP-1, MMP-3, and MMP-13 depended on the activation of the NF-kB and p38 MAPK pathways, whereas the increased production of TIMP-1 depended on the Erk1/2 pathway [10].

In general, cartilage degradation is mediated by MMP family, which is indeed well accepted [24]. High level of MMPs is regarded as one of the main expressions of OA [25, 26], as well as low level of aggrecan and type II collagen [27]. Proinflammatory cytokines induce expression of matix-degrading enzymes that degrade cartilage matrix and aggravate OA [28]. In addition, these cytokines downregulate the expression of genes involved in maintenance of cartilage matrix (like aggrecan and type II collagen), and promote hypertrophy and apoptosis of chondrocytes [29]. In the present study, up regulation of MMP-2, 3, 9, 13 and aggrecanses (adamts 4/5), down regulation of Collagen II and aggrecan caused by IL-18 stimulation were observed by qRT-PCR and Western blot, thus we confirmed the inflammatory effect of IL-18.

Besides NF-kB, PI3K, p38 MAPK and Erk1/2 pathways $[10,30,31]$, the regulation of chondrocytespecific proteins and matix-degrading enzymes is also related to some other pathways. Ma B et al reported that the expression of MMPs could be regulated by Wnt/ $\beta$-catenin pathway [32]. A study by $\mathrm{Xu} \mathrm{HG}$ et al demonstrated that intermittent cyclic mechanical tension loading led to $\mathrm{Wnt} / \beta$-catenin signaling activation and loss of chondrogenic phonetype of chondrocytes [17]. Another recent study performed by Cheleschi $\mathrm{S}$ et al reported that hydrostatic pressure restores the expression levels of some miRNAs and downregulates some matixdegrading enzymes via the Wnt/ $\beta$-catenin pathway [16]. Study performed by Lin AC et al demonstrated that Hedgehog signaling regulates normal chondrocyte and differentiation, and they found Hedgehog signaling is activated in osteoarthritis [18]. Meanwhile, our previous studies also indicated that several drugs could provide chondroprotective effects on OA by inhibiting Wnt/ $\beta$ catenin and Hedgehog pathways $[33,34]$. In this study, we investigated the role of Wnt/ $\beta$-catenin and Hedgehog pathways in the proinflammatory effect of IL-18 on OA. Results of Western blot showed increased level of $\beta$-catenin, GSK-3 $\beta$, DKK-1, IHH, SHH, Gli-2, ptch1, and Smo in whole cell caused by IL-18 stimulation, which
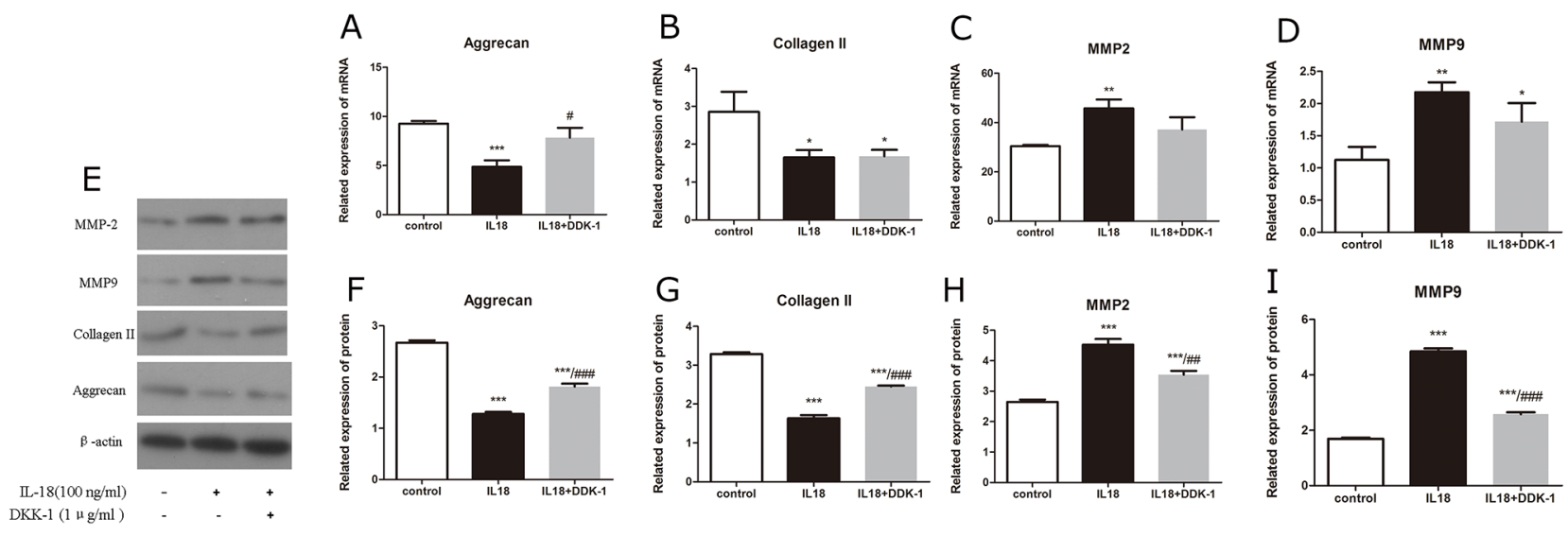

Figure 6: DKK-1 down-regulates IL-18-induced expression of matrix-degrading encymes. Chondrocytes of IL-18+DKK-1 group were pre-treated with DKK-1 $(1 \mu \mathrm{g} / \mathrm{ml})$ for 1 hour, followed with $24 \mathrm{~h} \mathrm{IL-18} \mathrm{stimulation} \mathrm{(100ng/ml).} \mathrm{Chondrocytes} \mathrm{of} \mathrm{IL-18} \mathrm{group}$ were treated with $100 \mathrm{ng} / \mathrm{ml}$ IL-18 for 24 hours. The expression of chondrocyte-specific proteins and matix-degrading enzymes, MMP-2, MMP-9, aggrecan, and Collagen II were evaluated by Real-Time PCR at mRNA level (A-D) and by Western blot at protein level (E-I). Significance was calculated by a one-way ANOVA with a post hoc Tukey's multiple comparisons test. ${ }^{*} p<0.05,{ }^{* *} \mathrm{p}<0.01,{ }^{* * *} \mathrm{p}<0.001$ versus control group. ${ }^{*} \mathrm{p}<0.05,{ }^{\# \#} \mathrm{p}<0.01,{ }^{* \# \#} \mathrm{p}<0.001$ versus $100 \mathrm{ng} / \mathrm{ml}$ IL-18 treated group 

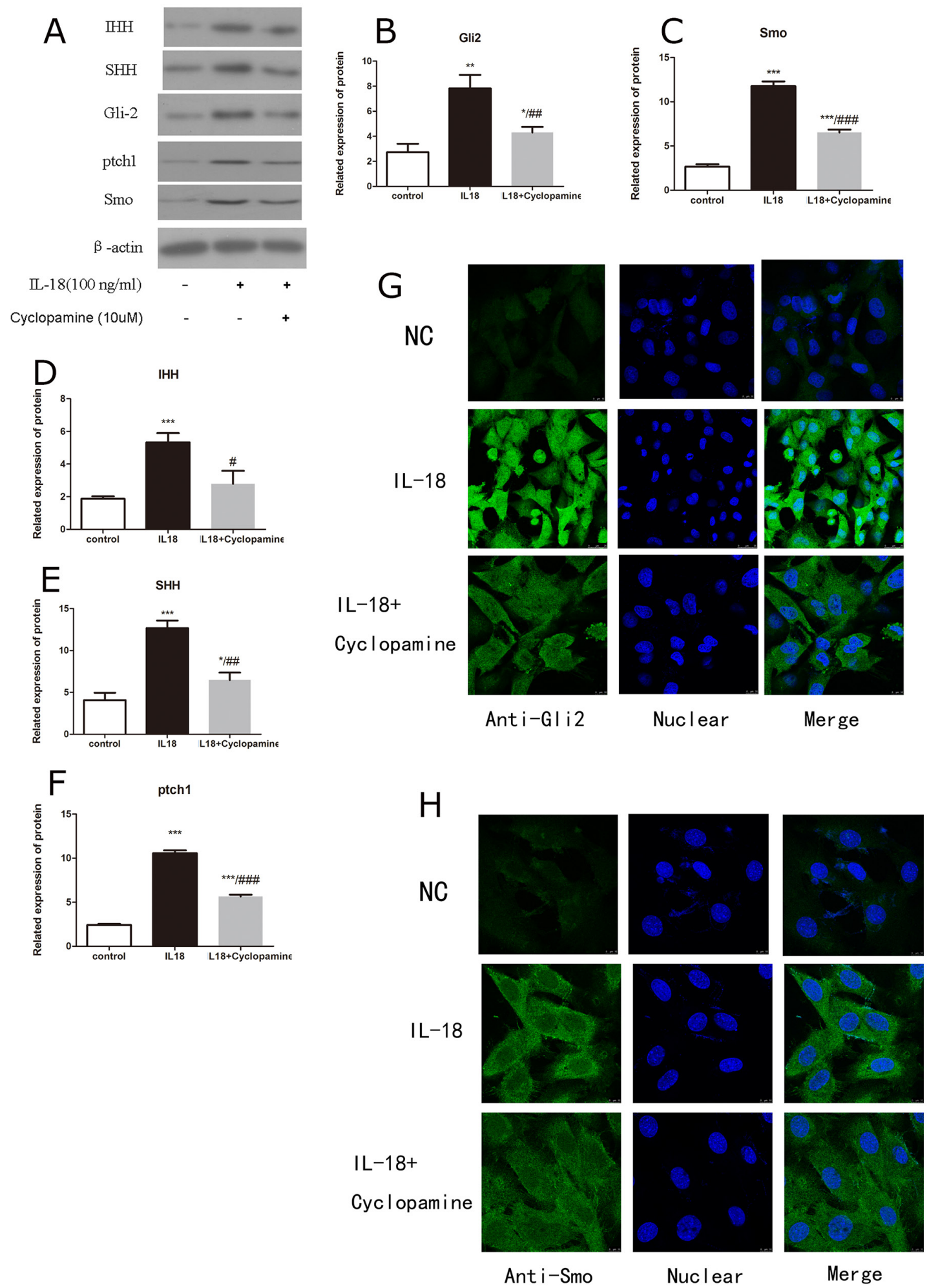

Figure 7: Cyclopamine worked as a potent antagonist of Hedgehog pathway. Chondrocytes of IL-18+ Cyclopamine group were pre-treated with Cyclopamine $(10 \mu \mathrm{M})$ for 1 hour, followed with $24 \mathrm{~h}$ IL-18 stimulation (100ng/ml). Chondrocytes of IL-18 group were treated with 100ng/ml IL-18 for 24 hours. The level of IHH (D), SHH (E), ptch1 (F), Gli2 (B) and Smo (C) in total exact was investigated by Western blot (A). Significance was calculated by a one-way ANOVA with a post hoc Tukey's multiple comparisons test. ${ }^{*} \mathrm{p}<0.05,{ }^{* *} \mathrm{p}<0.01,{ }^{* * *} \mathrm{p}<0.001$ versus control group. ${ }^{\#} \mathrm{p}<0.05,{ }^{\#} \mathrm{p}<0.01,{ }^{\# \#} \mathrm{p}<0.001$ versus $100 \mathrm{ng} / \mathrm{ml}$ IL-18 treated group. The location and concentration of Gli2 (G) and Smo (H) was visualized using immunofluorescence analysis. 
Table 1: Primers used for Real-Time PCR

Primer Sequences (5'-3')

\begin{tabular}{lllc}
\hline Gene $^{*}$ & Forward & Reverse & Amplicon Size (bp) \\
\hline Rat MMP2 & GTACTGGACCCACGCCTACACT & CGCCAAATAAACCGATCCTTGA & 124 \\
Rat MMP9 & GCAAACCCTGCGTATTTCCAT & GATAACCATCCGAGCGACCTTT & 73 \\
$\begin{array}{l}\text { Rat Collagen } \\
\text { II }\end{array}$ & GAGTGGAAGAGCGGAGACTACTG & GTCTCCATGTTGCAGAAGACTTTCA & 83 \\
Rat aggrecan & CTAGCTGCTTAGCAGGGATAACG & GATGACCCGCAGAGTCACAAAG & 110 \\
Rat GAPDH & GAAGGTCGGTGTGAACGGATTTG & CATGTAGACCATGTAGTTGAGGTCA & 127 \\
\hline
\end{tabular}

${ }^{*}$ Rat means species is rat

means the inflammatory effect of IL-18 may be related to $\mathrm{Wnt} / \beta$-catenin and Hedgehog pathways. As $\beta$-catenin and Gli-2 were transported into cell nucleus when the pathways were activated, the amounts of $\beta$-catenin and Gli-2 in cell nucleus were investigated in addition to confirm the activation of Wnt/ $\beta$-catenin and Hedgehog pathways, and results of Western blot showed increased amounts of both $\beta$-catenin and Gli-2 in cell nucleus. In order to confirm the connection between the pathways and inflammatory effect of IL-18 on chondrocyte, we ran plenty of pathway inhibition tests. DKK-1 that was used as inhibitor of $\mathrm{Wnt} / \beta$-catenin pathway downregulated MMP-2 and MMP-9 and upregulated aggrecan in the presence of IL-18 according to the results of qRT-PCR and Western blot. We also noticed that the results of qRTPCR and Western blot of Collagen II was a little different (Figure 6). According to a study published in 2015, the
Wnt signaling orchestrates a rich post-transcriptional regulatory function [35], so the different expression level of mRNA and protein of Collagen II may be related to the post-transcriptional regulatory function of Wnt signaling. On the other hand, we used Cyclopamine as blocker of Hedgehog signaling. Resutlts showed that Cyclopamine treatment decreased MMP-2 and MMP-9 and increased Collagen II and aggrecan in the presence of IL-18, and Cyclopamine also downregulated the amount of Gli-2 in cell nucleus. As the relationship and distribution of IL18Rs and the effect of IL-18 on cell proliferation and apoptosis have been investigated in other studies, so we didn't run similar researches $[8,11]$

Collectively, our results demonstrated that Wnt/ $\beta$ catenin and Hedgehog pathways were involved in the inflammatory effect of IL-18 in vitro. These findings would help further understanding of IL-18 action.

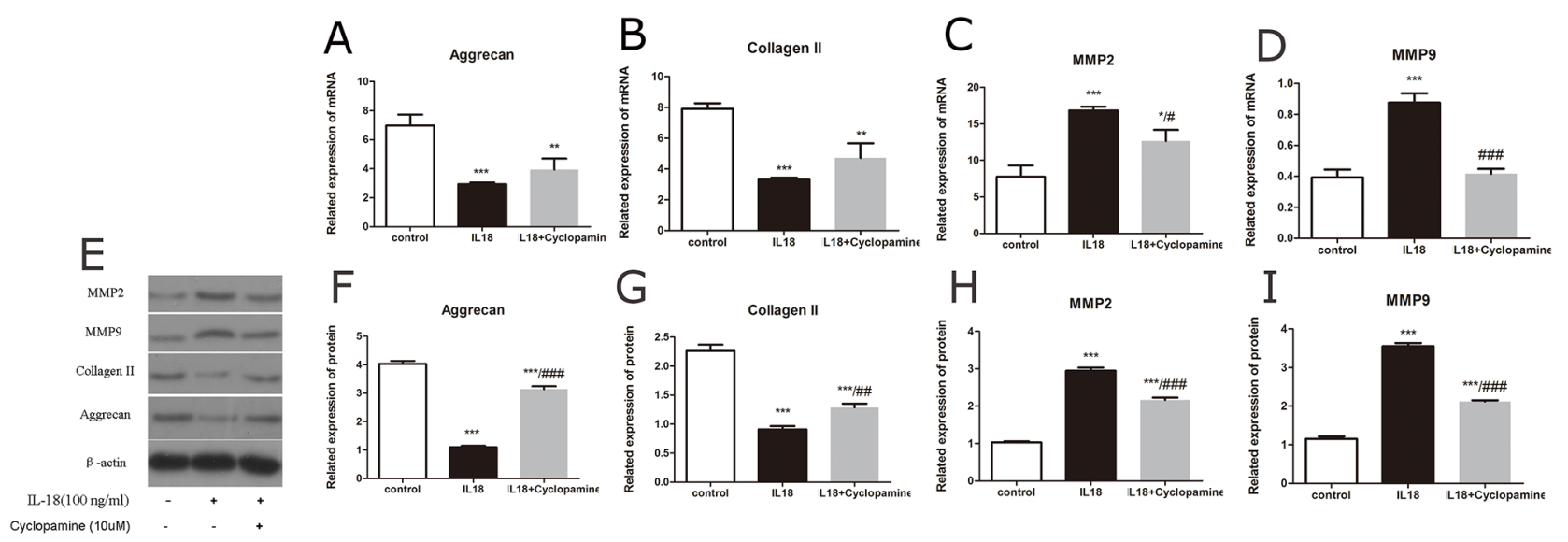

Figure 8: Cyclopamine down-regulates IL-18-induced expression of matrix-degrading encymes. Chondrocytes of IL-18+ Cyclopamine group were pre-treated with Cyclopamine $(10 \mu \mathrm{M})$ for 1 hour, followed with $24 \mathrm{~h}$ IL-18 stimulation $(100 \mathrm{ng} / \mathrm{ml})$. Chondrocytes of IL-18 group were treated with 100ng/ml IL-18 for 24 hours. The expression of chondrocyte-specific proteins and matix-degrading enzymes, MMP-2, MMP-9, aggrecan, and Collagen II were evaluated by Real-Time PCR at mRNA level (A-D) and by Western blot at protein level (E-I). Significance was calculated by a one-way ANOVA with a post hoc Tukey's multiple comparisons test. "p $<0.05$, ${ }^{* *} \mathrm{p}<0.01,{ }^{* * *} \mathrm{p}<0.001$ versus control group. ${ }^{\#} \mathrm{p}<0.05,{ }^{\# *} \mathrm{p}<0.01,{ }^{\# \#} \mathrm{p}<0.001$ versus $100 \mathrm{ng} / \mathrm{ml}$ IL-18 treated group 


\section{MATERIALS AND METHODS}

\section{Materials}

Recombinant rat IL-18 and rat DKK- 1 as Wnt/ $\beta$ catenin inhibitor were purchased from R\&D Systems, Abingdon, UK. Dulbecco's Modified Eagle's Medium (DMEM), penicillin and streptomycin, fetal bovine serum (FBS), $0.25 \%$ trypsin were obtained from Gibco RRL, Grand Island, NY, USA. Cyclopamine as Hedgehog inhibitor and collagenase II were purchased from SigmaAldrich, St, Louis, MO, USA.

\section{Cell culture and treatment}

This study was approved by the Ethics Committee of the $2^{\text {nd }}$ Affiliated Hospital, School of medicine, Zhejiang University, Hangzhou, China. Four-week-old Sprague Dawley rats were sacrificed, and cartilage harvested from the knee joints was digested immediately with $0.25 \%$ pancreatic enzymes for $30 \mathrm{~min}$ to remove other tissues and cells. Then, the cartilage fragments were digested with $0.2 \%$ collagenase II on a horizontal shaker at $37^{\circ} \mathrm{C}$ for 4 hours to isolate the cells in cartilage. The chondrocytes were grown in DMEM with $10 \% \mathrm{FBS}, 100 \mathrm{U} / \mathrm{mL}$ penicillin, and $100 \mathrm{mg} / \mathrm{mL}$ streptomycin at $37^{\circ} \mathrm{C}$ with $5 \% \mathrm{CO} 2$.. Cells were seeded in six-well plates for analysis. When studying the inflammatory effect of IL-18, Subconfluent cells were treated with IL-18 in various concentrations $(0 \mathrm{ng} / \mathrm{ml}$ as control, $1 \mathrm{ng} / \mathrm{ml}, 10 \mathrm{ng} / \mathrm{ml}, 100 \mathrm{ng} / \mathrm{ml}$ ) for 24 hours before analysis. According to the results, we chose $100 \mathrm{ng} / \mathrm{ml}$ as the concentration of IL-18 in the following pathway inhibition tests. In pathway inhibition tests, subconfluent cells were pretreated with DKK-1 $(1 \mu \mathrm{g} / \mathrm{ml})$ or Cyclopamine $(10 \mu \mathrm{m})$ for $1 \mathrm{~h}$ then incubated with IL-18 (100ng/ml) for 24 hours before analysis.

\section{RNA extraction and Real-Time PCR}

TRIzol reagent (Invitrogen, Carlsbad, CA, USA) was used to isolate RNA from chondrocytes accordingly. Total RNA was used to synthesize cDNA by reverse transcription (cDNA synthesis kit, Takara). Power SYBR Green PCR Master Mix (Applied Biosystems) was used for real-time PCR. The expression of MMP2, MMP9, Collagen II, and aggrecan was analyzed using primer sequences listed in Table 1. Primer sequences listed in Supplementary Table 1 were used to analyze the mRNA level of MMP3, MMP13, Adamts4 and Adamts5. For real-time PCR, $20 \mu \mathrm{L}$ reaction mixture contained SYBR Green and each primer. PCR was set using StepOnePlus system (Applied Biosystems). The program included 1 cycle of denaturation at $95^{\circ} \mathrm{C}$ for 1 min and 40 cycles of denaturation at $95^{\circ} \mathrm{C}$ for $15 \mathrm{~s}$, primer annealing, and extension at $63^{\circ} \mathrm{C}$ for $25 \mathrm{~s}$, followed by melt curve analysis. Data were analyzed for fold difference using formula: $2^{\text {(CT GAPDH-CT targeted gene) }} \times 10$.

\section{Western blot analysis}

After treatment, whole cell extract was prepared using radioimmunoprecipitation assay (RIPA) containing protease and phosphatase inhibitors according to the manufacturer's protocol. Nuclear and cytosolic extracts were prepared using NE-PER Nuclear and Cytoplasmic Extraction Reagents (Pierce Biotechnology, Rockford, IL, USA). Equal amounts of cell extract were separated by $10 \%$ SDS-PAGE, and electro-transferred to polyvinylidene difluoride membranes. After blocked with $7 \%$ nonfat milk for 2 hours, the membranes were blotted with primary antibodies at $4{ }^{\circ} \mathrm{C}$ overnight, incubated for 1 hour with secondary antibody. In this study, antibodies (Ab) against $\beta$-catenin (Abcam ab32572), glycogen synthase kinase-3 beta (GSK-3 $\beta$, Abcam ab93926), DKK-1 (Abcam ab109416), india hedgehog (IHH, Abcam ab52919), sonic hedgehog (SHH, Abcam ab202342), GLI family zinc finger 2 (Gli-2, Santa cruz SC-28674), $\beta$-actin (Santa Cruz SC-47778), patch 1 (ptch1, Santa cruz SC-9016), TATA-box binding protein (TBP, Abcam ab197874), and smoothened (Smo, Santa cruz SC-13943) were used. Finally, signals were detected using West Dura Extended Duration Substrate with exposure to X-ray film.

\section{Immunofluorescence microscopy}

Coexpression of key proteins of $\mathrm{Wnt} / \beta$-catenin and Hedgehog pathways was carried out using fluorochromeconjugated antibodies. Chondrocytes cultured on glass coverslips were fixed in $4 \%$ paraformaldehyde for 10 $\mathrm{min}$, and permeabilized for $5 \mathrm{~min}$ with $0.1 \% \mathrm{v} / \mathrm{v}$ Triton $\mathrm{X}-100$. Cells were incubated with primary antibody for $4 \mathrm{~h}$, washed, and then incubated with fluorochrome-conjugated secondary $\mathrm{Ab}$ for $2 \mathrm{~h}$ in the dark. Coverslips were mounted onto glass slides using DAPI-containing mounting medium.

\section{Statistical analysis}

All experiments were repeated three times. All the data in figures are presented as a grouped column scatter of three repeats with diplayed mean. Statistical differences were performed with SPSS 12.0 version. Oneway ANOVA with a subsequent post hoc Tukey's test was used for multiple comparisons. $\mathrm{P}<0.05$ is considered to be significant with statistical meaning.

\section{Abbreviations}

IL-18, Interleukin 18

DKK-1, Dickkopf-1

FBS, fetal bovine serum

MMP, matrix metalloproteinase

$\mathrm{Ab}$, antibodies

GSK-3 $\beta$, glycogen synthase kinase- 3 beta

$\mathrm{IHH}$, india hedgehog

SHH, sonic hedgehog 
Gli-2, GLI family zinc finger 2

ptch 1, patch 1

TBP, TATA-box binding protein

Smo, smoothened

$\mathrm{NF}-\mathrm{kB}$, nuclear factor-kB

MAPK, mitogen activated protein kinase

OA, Osteoarthritis

\section{Statements}

Jiapeng Bao and Chiyuan Ma contributed equally to this study.

All authors listed were involved in the study and preparation of the manuscript.

All conference has been list at the end of the paper with mark in the manuscript.

All data supporting the conclusions are included in this article.

The protocols of study were approved by the Ethics Committee of the 2nd affiliated hospital, School of Medicine, Zhejiang University, Hangzhou, China.

\section{Author contributions}

All authors listed have made substantial contributions to the study. LD, SY, JB and CM took part in the designing of the experiments, contributed reagents, materials and analysis tools. $\mathrm{JB}, \mathrm{CM}, \mathrm{YX}$ and JS run the experiments. JB and $\mathrm{CM}$ wrote the manuscript. JB and $\mathrm{YX}$ also participated in the analyzing of the data. All authors read and approved the final manuscript.

\section{CONFLICTS OF INTEREST}

All authors have no conflicts of interest.

\section{FUNDING}

This study is funded by National Natural Science Foundation of China (NO. 81572173, 81371996, 81401824) and Natural Science Foundation of Zhejiang Province (NO. LQ17H060002, Y14H060014).

\section{REFERENCES}

1. Bijlsma JW, Berenbaum F, Lafeber FP. Osteoarthritis: an update with relevance for clinical practice. Lancet. 2011; 377: 2115-26.

2. Wojdasiewicz P, Poniatowski LA, Szukiewicz D. The role of inflammatory and anti-inflammatory cytokines in the pathogenesis of osteoarthritis. Mediators Inflamm. 2014; 2014: 561459.

3. Chen AC, Temple MM, Ng DM, Verzijl N, DeGroot J, TeKoppele JM, Sah RL. Induction of advanced glycation end products and alterations of the tensile properties of articular cartilage. Arthritis Rheum. 2002; 46: 3212-7.

4. Goldring MB, Otero M. Inflammation in osteoarthritis. Curr Opin Rheumatol. 2011; 23: 471-8.
5. Tetlow LC, Adlam DJ, Woolley DE. Matrix metalloproteinase and proinflammatory cytokine production by chondrocytes of human osteoarthritic cartilage: associations with degenerative changes. Arthritis Rheum. 2001; 44: 585-94.

6. Ghayur T, Banerjee S, Hugunin M, Butler D, Herzog L, Carter A, Quintal L, Sekut L, Talanian R, Paskind M, Wong W, Kamen R, Tracey D, et al. Caspase-1 processes IFNgamma-inducing factor and regulates LPS-induced IFNgamma production. Nature. 1997; 386: 619-23.

7. Dinarello CA. Overview of the interleukin-1 family of ligands and receptors. Semin Immunol. 2013; 25: 389-93.

8. Olee T, Hashimoto S, Quach J, Lotz M. IL-18 is produced by articular chondrocytes and induces proinflammatory and catabolic responses. J Immunol. 1999; 162: 1096-100.

9. Udagawa N, Horwood NJ, Elliott J, Mackay A, Owens J, Okamura H, Kurimoto M, Chambers TJ, Martin TJ, Gillespie MT. Interleukin-18 (interferon-gamma-inducing factor) is produced by osteoblasts and acts via granulocyte/ macrophage colony-stimulating factor and not via interferon-gamma to inhibit osteoclast formation. J Exp Med. 1997; 185: 1005-12.

10. Dai SM, Shan ZZ, Nishioka K, Yudoh K. Implication of interleukin 18 in production of matrix metalloproteinases in articular chondrocytes in arthritis: direct effect on chondrocytes may not be pivotal. Ann Rheum Dis. 2005; 64: 735-42.

11. John T, Kohl B, Mobasheri A, Ertel W, Shakibaei M. Interleukin-18 induces apoptosis in human articular chondrocytes. Histol Histopathol. 2007; 22: 469-82.

12. Joosten LA, Smeets RL, Koenders MI, van den Bersselaar LA, Helsen MM, Oppers-Walgreen B, Lubberts E, Iwakura Y, van de Loo FA, van den Berg WB. Interleukin-18 promotes joint inflammation and induces interleukin1-driven cartilage destruction. Am J Pathol. 2004; 165: 959-67.

13. Joosten LA, van De Loo FA, Lubberts E, Helsen MM, Netea MG, van Der Meer JW, Dinarello CA, van Den Berg WB. An IFN-gamma-independent proinflammatory role of IL-18 in murine streptococcal cell wall arthritis. J Immunol. 2000; 165: 6553-8.

14. Kawaguchi H. Regulation of osteoarthritis development by Wnt-beta-catenin signaling through the endochondral ossification process. J Bone Miner Res. 2009; 24: 8-11.

15. Dell'accio F, De Bari C, Eltawil NM, Vanhummelen P, Pitzalis C. Identification of the molecular response of articular cartilage to injury, by microarray screening: Wnt-16 expression and signaling after injury and in osteoarthritis. Arthritis Rheum. 2008; 58: 1410-21.

16. Cheleschi S, De Palma A, Pecorelli A, Pascarelli NA, Valacchi G, Belmonte G, Carta S, Galeazzi M, Fioravanti A. Hydrostatic Pressure Regulates MicroRNA Expression Levels in Osteoarthritic Chondrocyte Cultures via the Wnt/beta-Catenin Pathway. Int J Mol Sci. 2017; 18.

17. Xu HG, Zheng Q, Song JX, Li J, Wang H, Liu P, Wang J, Wang CD, Zhang XL. Intermittent cyclic mechanical tension 
promotes endplate cartilage degeneration via canonical Wnt signaling pathway and E-cadherin/beta-catenin complex cross-talk. Osteoarthritis Cartilage. 2016; 24: 158-68.

18. Lin AC, Seeto BL, Bartoszko JM, Khoury MA, Whetstone H, Ho L, Hsu C, Ali SA, Alman BA. Modulating hedgehog signaling can attenuate the severity of osteoarthritis. Nat Med. 2009; 15: 1421-5.

19. Glinka A, Wu W, Delius H, Monaghan AP, Blumenstock C, Niehrs C. Dickkopf-1 is a member of a new family of secreted proteins and functions in head induction. Nature. 1998; 391: 357-62.

20. Cooper MK, Porter JA, Young KE, Beachy PA. Teratogenmediated inhibition of target tissue response to $\mathrm{Shh}$ signaling. Science. 1998; 280: 1603-7.

21. Peng CZ, Cao JM, Xiao T, Peng C, Yang HB, Chen X, Fang JZ. Concentration of IL-18 and PGE2 in synovial fluid in patients with osteoarthritis and its significance. [Article in Chinese]. Zhong Nan Da Xue Xue Bao Yi Xue Ban. 2006; 31: $862-5$.

22. Wang $\mathrm{Y}, \mathrm{Xu} \mathrm{D}$, Long L, Deng X, Tao R, Huang G. Correlation between plasma, synovial fluid and articular cartilage Interleukin-18 with radiographic severity in 33 patients with osteoarthritis of the knee. Clin Exp Med. 2014; 14: 297-304.

23. Denoble AE, Huffman KM, Stabler TV, Kelly SJ, Hershfield MS, McDaniel GE, Coleman RE, Kraus VB. Uric acid is a danger signal of increasing risk for osteoarthritis through inflammasome activation. Proc Natl Acad Sci U S A. 2011; 108: 2088-93.

24. Troeberg L, Nagase H. Proteases involved in cartilage matrix degradation in osteoarthritis. Biochim Biophys Acta. 2012; 1824: 133-45.

25. Zeng GQ, Chen AB, Li W, Song JH, Gao CY. High MMP-1, MMP-2, and MMP-9 protein levels in osteoarthritis. Genet Mol Res. 2015; 14: 14811-22.

26. Tchetverikov I, Lohmander LS, Verzijl N, Huizinga TW, TeKoppele JM, Hanemaaijer R, DeGroot J. MMP protein and activity levels in synovial fluid from patients with joint injury, inflammatory arthritis, and osteoarthritis. Ann Rheum Dis. 2005; 64: 694-8.
27. Matyas JR, Huang D, Chung M, Adams ME. Regional quantification of cartilage type II collagen and aggrecan messenger RNA in joints with early experimental osteoarthritis. Arthritis Rheum. 2002; 46: 1536-43.

28. Loeser RF. Molecular mechanisms of cartilage destruction: mechanics, inflammatory mediators, and aging collide. Arthritis Rheum. 2006; 54: 1357-60.

29. Kobayashi M, Squires GR, Mousa A, Tanzer M, Zukor DJ, Antoniou J, Feige U, Poole AR. Role of interleukin-1 and tumor necrosis factor alpha in matrix degradation of human osteoarthritic cartilage. Arthritis Rheum. 2005; 52: 128-35.

30. Sanchavanakit N, Saengtong W, Manokawinchoke J, Pavasant P. TNF-alpha stimulates MMP-3 production via PGE2 signalling through the NF-kB and p38 MAPK pathway in a murine cementoblast cell line. Arch Oral Biol. 2015; 60: 1066-74.

31. Yuan H, Yang P, Zhou D, Gao W, Qiu Z, Fang F, Ding S, Xiao W. Knockdown of sphingosine kinase 1 inhibits the migration and invasion of human rheumatoid arthritis fibroblast-like synoviocytes by down-regulating the PI3K/ AKT activation and MMP-2/9 production in vitro. Mol Biol Rep. 2014; 41: 5157-65.

32. Ma B, van Blitterswijk CA, Karperien M. A Wnt/betacatenin negative feedback loop inhibits interleukin-1induced matrix metalloproteinase expression in human articular chondrocytes. Arthritis Rheum. 2012; 64: 2589-600.

33. Zhou X, Li W, Jiang L, Bao J, Tao L, Li J, Wu L. Tetrandrine Inhibits the Wnt/ beta -Catenin Signalling Pathway and Alleviates Osteoarthritis: An In Vitro and In Vivo Study. Evid Based Complement Alternat Med. 2013; 2013: 809579.

34. Zhou X, Lin X, Xiong Y, Jiang L, Li W, Li J, Wu L. Chondroprotective effects of palmatine on osteoarthritis in vivo and in vitro: A possible mechanism of inhibiting the Wnt/beta-catenin and Hedgehog signaling pathways. Int Immunopharmacol. 2016; 34: 129-38.

35. Koch S, Acebron SP, Herbst J, Hatiboglu G, Niehrs C. Posttranscriptional Wnt Signaling Governs Epididymal Sperm Maturation. Cell. 2015; 163: 1225-36. 